\title{
GEORREFERENCIAMENTO DE MAPAS HISTÓRICOS NO AUTOCAD MAP 3D
}

\section{GEOREFERENCING OF HISTORICAL MAPS IN AUTOCAD MAP 3D}

Vanessa Dagostim
Manenti
Engenheira Agrimensora,
Mestranda do Programa de
Pós-Graduação em Ciências
Ambientais, Universidade
do Extremo Sul Catarinense
- UNESC. E-mail:
vanessa.dagostim@hotmail.
com
Mônica Priscila Kravczik
Guglielmi
Geografia Licenciatura,
Universidade do Extremo
Sul Catarinense - UNESC. E-
mail:
kravczykmonica@gmail.com
Ambientais, Universidade
do Extremo Sul Catarinense
- UNESC. E-mail:
Iadwig@unesc.net
Professor do Ladwig

\section{RESUMO}

As geotecnologias, além de ter tornado mais ágil e seguro o processo de coleta de dados espaciais, também possuem grande aplicabilidade na reutilização de mapas antigos e mapas históricos em formato analógico. O processo de digitalização é etapa importante, pois permite a transformação do formato analógico (papel) para o formato digital. Todavia diversas dificuldades estão associadas, como as inconsistências encontradas nos mapas, a desatualização do sistema de coordenadas utilizado, situação de conservação, entre outros. O estudo tem como objetivo realizar o georreferenciamento de um mapa histórico por meio da ferramenta Rubbersheet do software Autocad Map 3D, analisar os erros obtidos e descrever detalhadamente a metodologia utilizada.

Palavras chaves: Georreferenciamento, Autocad, Rubbersheet.

\section{ABSTRACT}

Geotechnologies, in addition to make the process of spatial data collection more agile and secure, also have great applicability in the reuse of old maps and historical maps in analog format. The process of scanning is perhaps the most important step as it allows the transformation of the analog format (paper) into the digital format. However, several difficulties are associated, such as the inconsistencies found in the maps, the outdated coordinate system used, the conservation situation, among others. The present study, therefore, aims to present the georeferencing of a historical map through the Rubbersheet tool of Autocad Map 3D, analyze the errors obtained and describe in detail the methodology used for this purpose.

Keywords: Georeferencing, Autocad, Rubbersheet 


\section{INTRODUÇÃO}

Desde os primórdios da existência humana as representações espaciais, seja por meio de símbolos ou desenhos, sempre estiveram presentes, sendo utilizadas principalmente para demarcar o território ocupado. Neste contexto surge a Cartografia, definida pelo IBGE (1998, apud ACI, 1996, p. 10) como um conjunto de estudos e operações científicas, técnicas e artísticas que tem como objetivo a elaboração de mapas, cartas e outras formas de expressão ou representação de objetos, elementos, ambientes físicos e socioeconômicos, tendo por base os resultados de observações diretas ou da análise de documentação.

A medida que a Cartografia evoluiu, as técnicas de representação e os recursos se tornaram cada vez mais modernos, aumentando assim a possibilidade de representar os fenômenos de forma mais detalhada e precisa. Silva e Carvalho (2011, apud Schimiguel, 2004, p. 86) afirmam que as geotecnologias "têm tornado mais ágil e seguro o processo de coleta de dados espaciais; a elaboração de um produto cartográfico ou até mesmo a extração de novas informações espaciais sobre um determinado produto cartográfico já criado". De fato, enquanto as primeiras representações embasavam-se na posição dos astros e na utilização de instrumentos simples, atualmente os produtos são gerados, além da convencional topografia, por meio da utilização de imagens de satélites e Sistemas Globais de Posicionamento por Satélite (GNSS).

Além da possibilidade de utilização para a geração de novos produtos, as geotecnologias também possuem grande aplicabilidade na reutilização de mapas antigos e mapas históricos em formato analógico, o que é significativo no caso de estudos temporais e atualização cartográfica, por exemplo. Neste processo, a digitalização é a etapa mais importante, pois permite a transformação do formato analógico (papel) para o formato digital. Contudo, diversas dificuldades estão associadas a essa metodologia, como as inconsistências encontradas nos mapas, a desatualização do sistema de coordenadas utilizado, situação de conservação, entre outros.

Diante do exposto, o presente estudo tem como objetivo ilustrar o georreferenciamento de um mapa histórico por meio do software Autocad Map 3D e descrever as etapas para tal fim, podendo servir como base para outros trabalhos da mesma natureza.

\section{REFERENCIAL TEÓRICO}

\subsection{Mapas históricos e mapas antigos}

Atualmente, para que uma representação seja considerada um mapa, é primordial a presença de alguns elementos básicos que complementem a representação cartográfica, como a indicação do Norte (geográfico, magnético ou de quadrícula); escala (gráfica e/ou numérica); coordenadas 
referenciadas a um sistema geográfico ou geodésico; altitudes referenciadas a uma origem (datum) vertical; sistema de projeção; entre outros. No passado, contudo, devido à constante evolução do conhecimento e das técnicas de representação, nem sempre os mapas possuíam tais elementos. O que os caracterizava, segundo Corrêa $(2008$, p. 23) é que eles representavam fenômenos em suas posições, senão absolutas, relativas à posição de outros fenômenos. Era esse posicionamento gráfico (ou geográfico) dos fenômenos da Terra conhecida que auxiliava, por exemplo, nas grandes navegações marítimas.

Algumas variáveis, como a forma e a cor, variando em tonalidade e saturação, muito expressivas na caracterização dos mapas de antigamente, ainda hoje permanecem nas representações contemporâneas.

Portanto, ao se analisar um mapa antigo ou um mapa histórico e ao se tentar comparar mapas de diferentes épocas, é tarefa difícil integrar os dados de um e outro mapa com o mínimo de distorção possível, visto que o estágio do entendimento tecnológico e científico na época da criação de cada mapa varia ao longo do tempo.

Algumas incertezas, porém, giram entorno das definições de mapa antigo e mapa histórico, devido estes conceitos não serem consolidados. Por este motivo considerar-se-á a definição dada por Corrêa (2008), o qual considera mapa antigo como sendo aquele publicado em épocas remotas, sendo esta antiguidade um aspecto relativo, e que sofrem atualização constante. Quanto ao mapa histórico, ele próprio pode ser um documento de tamanha relevância, talvez pelas técnicas utilizadas ou pelos fatos ou época que represente, e, por este motivo, é considerado histórico.

De maneira geral, além de desvendar técnicas utilizadas num passado remoto e de representar toda uma história que remete às mais variadas regiões geográficas, Rumsey e Williams (2002, p. 1, apud Corrêa 2008, p. 24) reforçam que "os mapas guardam as informações geográficas que são fundamentais para a reconstrução de lugares do passado". Além de manter informações que não são mantidas de forma escrita, como nomes de limites ou lugares, que foram modificadas ou eliminadas ao longo do tempo.

\subsection{Digitalização e erros inerentes}

Os avanços tecnológicos atuais, especificamente na computação gráfica, causaram e estão causando cada dia mais alterações significativas na Cartografia. Desta forma, todo o processo cartográfico auxiliado pelo computador vem sendo transformado e melhorado, de forma que o produto final não seja mais um mapa impresso numa folha de papel, mas sim um arquivo digital com precisão antes inimaginável. 
Seja para o planejamento urbano ou simplesmente para a extração de informações, no processo de coleta de dados dos mapas históricos é essencial que se tenha conhecimento dos procedimentos e dos tratamentos a serem tomados para que a informação extraída tenha o menor erro possível. No caso específico dos mapas analógicos, para que seja possível obter as informações, é primordial que os mapas passem por um processo de digitalização (escaneamento), que de acordo com Scarim e Teixeira (1994, apud Nero, 2000, p. 32), “corresponde à conversão de pontos e linhas de um mapa convencional para um formato compatível com a utilização em meio computacional". As técnicas para a realização deste processo podem ser feitas pelos métodos a seguir.

\subsubsection{Digitalização via mesa}

É um processo manual executado por meio de um mouse e uma mesa digitalizadora, cujo principio baseia-se na correspondência entre pontos de controle do mapa e pontos definidos na mesa. Com a carta georreferenciada, é possível vetorizar as feições de interesse por meio de um cursor.

De acordo com Oliveira (2005, p. 29), os pontos são capturados acionando-se uma única vez o mouse, já as retas, digitalizando-se o primeiro e último ponto. As curvas, arrastando-se o mouse seguindo seu contorno e finalmente os polígonos traçando várias retas consecutivas até o retorno ao ponto inicial.

\subsubsection{Digitalização via scanner}

É o processo de conversão dos arquivos em meio analógico para meio digital (formato raster), através de um scanner. Segundo Scarim e Teixeira (1994, apud Nero, 2000, p. 37), um scanner é um dispositivo ótico-eletrônico composto por uma fonte de luz e um sensor ótico (fotocélula), sendo seu princípio de funcionamento baseado na emissão de um feixe de luz, refletido pela superfície do mapa e registrado pelo sensor (geralmente o CCD, Charge Coupled Device).

A imagem gerada por este processo é composta por pixels, os quais possuem tamanho e cor, definindo-se assim uma resolução espacial e uma resolução radiométrica de acordo com o equipamento utilizado. A primeira, medida em pontos por polegada (dpi - dots per inch), está associada à capacidade de identificação dos elementos, já a segunda com o potencial que o scanner distingue diferentes tons de cinza no mapa. Todavia, independente desses fatores, é necessário lembrar que a imagem resultante pode conter "imperfeições devido a inúmeros fatores, desde 
Revista Tecnologia e Ambiente, v. 25, 2019, Criciúma, Santa Catarina/SC ISSN Eletrônico 2358-9426 e ISSN Impresso 1413-8131

defeitos de dobras e sujeiras no mapa original até falhas de dados espúrios [...]" (OLIVEIRA, 2005 p. 30).

\subsubsection{Erros inerentes ao processo}

A todo processo de digitalização associam-se erros que, conforme a fonte, podem ser classificados em inerentes e operacionais. Os primeiros se referem aos erros presentes nas fontes originais, enquanto os "erros operacionais são produzidos durante a entrada dos dados e manipulação digital nos programas” (FILHO, 1993, p. 351).

Dentre as possíveis falhas que podem ser encontradas, o somatório total dos vários erros inerentes ao processo pode ser expresso pela formulação teórica (eq. 1) definida por Oliveira (2005, p. 30):

Erro $=f(a)+f(m)+f(c)+f(d)+f(e)+f(p)+f(R M S)+f(p e)+f(i d)+f(a t)+f(h i)+I$

Onde:

$\mathrm{f}(\mathrm{a})$ = transformação entre superfície esférica e superfície plana;

$\mathrm{f}(\mathrm{m})=$ precisão projeção cartográfica e datum;

$\mathrm{f}(\mathrm{c})$ = interpretação cartográfica;

$\mathrm{f}(\mathrm{d})=$ erro do desenho devido ao tamanho das linhas;

$\mathrm{f}(\mathrm{e})=$ estabilidade do material (emendas dobras);

$\mathrm{f}(\mathrm{p})=$ erro no próprio processo de digitalização;

$\mathrm{f}(\mathrm{RMS})=$ erro médio quadrático;

$\mathrm{f}(\mathrm{pe})=$ precisão do equipamento (número de dígitos);

$\mathrm{f}(\mathrm{id})=$ idade do mapa;

$\mathrm{f}(\mathrm{at})=$ descrição dos atributos ligados aos dados espaciais;

$\mathrm{f}(\mathrm{hi})=$ dados históricos;

$\mathrm{I}=$ erros adicionais inexplicáveis.

\subsection{Técnicas para edição de imagens}

Embora se tome todos os cuidados necessários durante o processo de digitalização, dependendo da qualidade do produto cartográfico, existe a necessidade de um tratamento das imagens, de modo a melhorar a visualização do produto e facilitar a posterior vetorização. Entre as diversas técnicas, Scarim e Teixeira (1994, apud Nero, 2000, p. 42) destaca a eliminação de ruídos, aumento de contraste, aplicação de limiares, eliminação de informações marginais, mosaicagem de arquivos e complementação de linhas.

a) Eliminação de ruídos: etapa de edição que tem como objetivo eliminar os pixels com falta de informações da energia refletida em uma determinada área da imagem.

b) Aplicação de limiares: “é um processo no qual um determinado valor de brilho do pixel separa o que é considerado preto ou branco, convertendo o desenho em uma imagem binária". (NERO, 2000, p. 43) 
a) Eliminação de informações marginais: é a exclusão das informações que margeiam o desenho e que para efeitos de vetorização aumentam o tempo e o espaço de armazenamento. Por exemplo: cabeçalho, rodapé, legenda, entre outros.

b) Complementação de linhas: dependendo da sensibilidade do equipamento utilizado, as linhas digitalizadas podem apresentar algumas falhas no seu traçado, podendo gerar erros na vetorização. Para a resolução deste problema, pode-se utilizar ferramentas de edição matricial em softwares específicos.

c) Rotação da imagem: por vezes o produto gerado pela digitalização resulta rotacionado devido à incorreta inserção do mapa no equipamento ou até pelo próprio mapa não estar totalmente alinhado. Neste caso pode-se usar até os softwares mais simples que se consegue fazer a edição através do comando Rotate.

d) Mosaicagem: é a união de dois ou mais arquivos raster montados de forma a dar a impressão de que todo o conjunto é uma única imagem. De acordo com Nero (2000, p. 44) “é necessário realizar o georreferenciamento de cada uma das imagens, passando estas a estarem no mesmo sistema de coordenadas".

e) Suavização: processo que corresponde à eliminação de pixels nas bordas das linhas, o que melhora a qualidade da imagem e facilita a vetorização.

\subsection{Georreferenciamento de imagens}

Como o produto gerado por meio do processo de digitalização não resulta referenciado a um determinado sistema de coordenadas, para que seja possível a vetorização das feições em sua exata localização, é necessário que se faça o georreferenciamento do arquivo raster, viabilizando a utilização das imagens para a obtenção de informações.

Para Antunes (2000, apud Melo e Pacheco, 2004) o georreferenciamento é o processo que permite a imagem assumir propriedades cartográficas de sistema de projeção e respectivas coordenadas, é uma transformação entre coordenadas de pixels (linhas, colunas) para um sistema geográfico $(\mathrm{E}, \mathrm{N})$.

Já Gioielli (2006, p. 42, apud Giotto e Cardoso, 2010) afirmam que georreferenciar é tornar uma feição "referenciada espacialmente ao terreno por meio de sua localização, utilizando-se para tal um sistema de coordenadas conhecido, no qual a localização de um ponto da superfície da Terra pode ser identificada". Isto é feito através de parâmetros de transformação, os quais podem ser determinados por meio de pontos de controle, os quais devem ser precisamente identificados no produto digital. 
O procedimento de georreferenciamento utilizado neste estudo baseia-se na dissertação de mestrado de Lima (2013) que utiliza como modelo a transformação afim. De acordo com o mesmo, "transformação afim é o polinômio mais simples ou de primeiro grau, que possui 6 parâmetros de transformação (rotação, translação em X e Y, escalonamento em X e Y e a não ortogonalidade dos eixos de referência" (LIMA, 2013, p. 41). A vantagem deste modelo é a de manter o paralelismo e a retilineidade entre as linhas, além de preservar a convergência e as proporções entre as mesmas, mantendo assim a geometria das figuras. Portanto embora a imagem sofra translação, rotação e escalonamento, “a proporção, forma e a posição relativa dos pontos não se alteram” (LIMA, 2013, p. 41).

\subsection{Vetorização}

A vetorização em tela, comumente definida como heads-up, é um método manual de vetorização que consiste no desenho dos vetores sobre a imagem do mapa em tela com o uso de um mouse. É realizado em um software específico de desenho e ao final obtém-se um mapa vetorial com as feições separadas em layers. Tem a grande vantagem de permitir a visualização da sobreposição do vetor com a imagem, além de uma "visão detalhada dos pixels (através do zoom)" (NERO, 2000, p.47). É considerado o método mais indicado quando a qualidade do arquivo é baixa.

\section{METODOLOGIA}

Durante a explanação a seguir, procurou-se adaptar os procedimentos metodológicos ao objetivo a ser alcançado, sendo os mesmos delineados por intermédio da seguinte abordagem: seleção do mapa com bagagem histórica relevante; digitalização; inserção do arquivo raster no Autocad Map 3D; seleção dos pontos de controle e modelo de transformação; georreferenciamento e vetorização.

A primeira etapa do processo foi a seleção do mapa a ser georreferenciado. $O$ acervo disponível para escolha, com mais de 6.000 plantas em formato analógico, é pertencente à uma indústria carbonífera do município de Criciúma (SC). Para este trabalho se utilizará como exemplo a representação intitulada como “A Planta da Área do Manifesto n 289/36 e Lotes Coloniais Correspondentes", na escala 1:10.000, localizada no município de Urussanga (SC), devido a futura utilização da mesma pela empresa.

A digitalização da planta em papel vegetal, isto é o processo de conversão dos arquivos em meio analógico para o formato raster (jpg), se deu através do Scanner HP Designjet T1200 e durou cerca de 3 minutos. A fim de que o arquivo obtivesse uma qualidade razoável e não ocupasse muito espaço no armazenamento, a resolução pré-determinada foi de 200 dpi. 
Com a planta em formato digital, especificamente formato raster, a segunda etapa se caracterizou pela edição da mesma no programa de manipulação de imagem GIMP 2.8.14. Para que o processo de vetorização fosse facilitado, foram realizados os procedimentos de rotação e recorte através dos comandos Ferramentas - Ferramentas de transformação - Rotacionar/Cortar e aplicação de filtro através da sequência Cores - Automático - Equilíbrio de branco.

Após a edição e o salvamento da imagem, se passou à inserção da mesma no software Autocad Map 3D 2016. É importante ressaltar que a extensão Autocad Raster Design deve estar adicionada, pois é a mesma que permite a execução do georreferenciamento da planta e a visualização do Erro Médio Quadrático. O procedimento requer poucos passos e não apresenta dificuldades significativas, bastou executar em sequência: Map Drafting - Image - Insert.

Com a planta inserida no Autocad Map 2016, se passou à escolha dos pontos de controle, tais como "intersecções de quadrícula, que conforme teste realizado por Kleiner; Meneguette (1999), [...] pode ser de uns treze pontos, bem distribuídos na imagem” (NERO, 2000, p.48). Entretanto, optou-se por utilizar todos os pontos correspondentes às intersecções da grade, isto é, cerca de 90 pontos, possibilitando assim alcançar uma maior precisão.

Com os pontos de controle do arquivo de origem (raster) estabelecidos, se inseriu manualmente os pontos de destino, de acordo com as coordenadas definidas pelas intersecções da grade. Em seguida, com a ferramenta Rubbersheet (Método triangular), foi possível realizar o georreferenciamento e verificar o Erro Médio Quadrático (RMS), o qual é dado automaticamente pelo software.

A vetorização em tela, isto é o desenho dos vetores sobre a imagem do mapa em tela com o mouse, foi a última e mais simples etapa a ser executada. Após a definição dos layers, das cores, espessuras e tipologias de linha a serem utilizados, se iniciou o processo no próprio Autocad Map $3 \mathrm{D}$.

\section{APRESENTAÇÃO E ANÁLISE DOS DADOS}

$\mathrm{Na}$ sequência de todos os procedimentos citados anteriormente, obteve-se a planta digitalizada inserida em ambiente CAD e georreferenciada com a ferramenta Rubbersheet (Figura 2). Como a planta não indica a qual datum a mesma se refere e também por não ser objetivo deste trabalho, não se realizou a transformação do datum antigo para o atual oficial (SIRGAS 2000).

Figura 2 - Parte da planta georreferenciada com a ferramenta Rubbersheet 
Revista Tecnologia e Ambiente, v. 25, 2019, Criciúma, Santa Catarina/SC ISSN Eletrônico 2358-9426 e ISSN Impresso 1413-8131

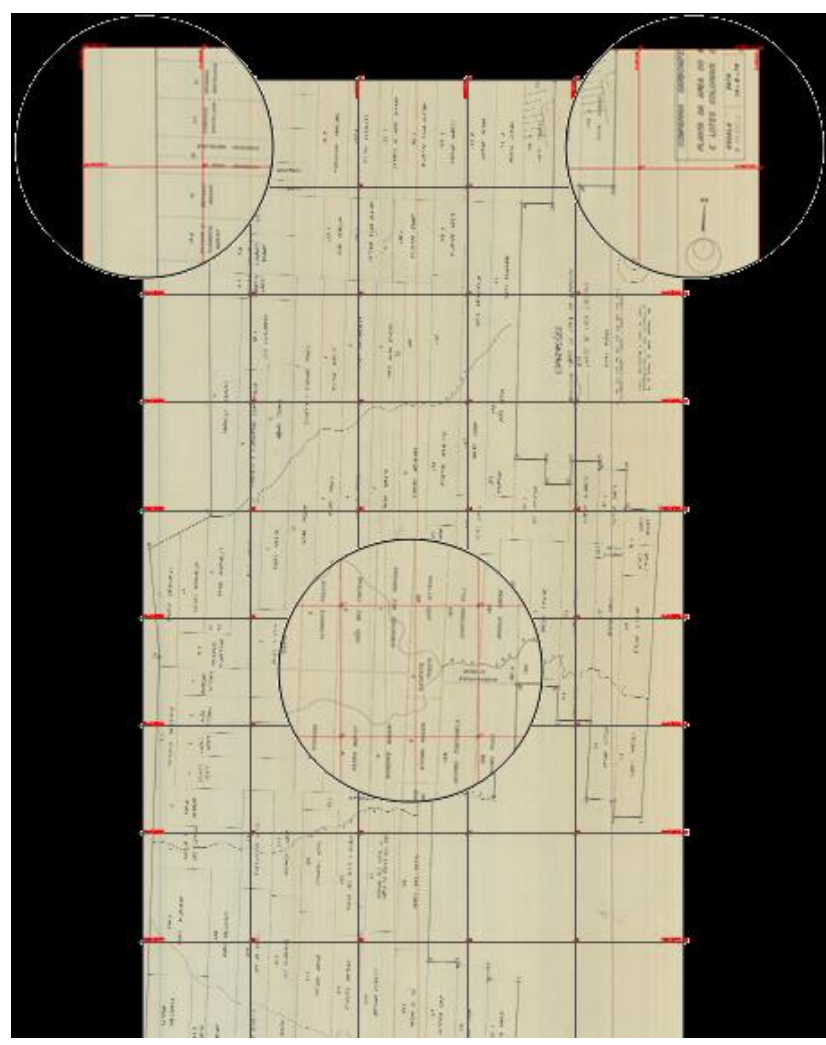

Fonte: Autor, 2016

Como toda transformação sempre acarreta erros, após a execução da ferramenta Rubbersheet, o software indicou em tela o RMS obtido com a utilização dos 90 pontos de controle, como ilustra a Figura 3 abaixo.

Figura 3 - RMS calculado pelo software

\begin{tabular}{|c|c|c|}
\hline \multicolumn{3}{|c|}{ A Rubbersheet - Set Control Points } \\
\hline \multicolumn{3}{|l|}{ Method } \\
\hline \multicolumn{3}{|l|}{ (2) Triangular } \\
\hline Polynomial & $\frac{A}{V} D$ & egree \\
\hline ID & Error & Source Point \\
\hline$\nabla 75$ & 0.0000000001164 & $651883.4821,6838150.6849$ \\
\hline V 76 & 0.0000000009313 & $653052.9427,6838143.502$ \\
\hline 目 77 & 0.0000000009313 & $654220.3633,6838140.867$ \\
\hline 78 & 0.0000000009385 & $648365.5374,6836992.5721$ \\
\hline 79 & 0.0000000009313 & $649526.4872,6836983.8800$ \\
\hline$\nabla 80$ & 0.0000000009313 & $650699.2310,6836976.294$ \\
\hline D 81 & 0.0000000009313 & $651880.5814,6836968.367$ \\
\hline 目 82 & 0.0000000009313 & $653047.3325,6836959.1025$ \\
\hline D 83 & 0.0000000009313 & $654215.1356,6836952.8760$ \\
\hline D 84 & 0.0000 & $648362.9555,6835812.2099$ \\
\hline D 85 & 0.0000 & $649524.0009,6835803.114$ \\
\hline V 86 & 0.0000 & $650695.5653,6835796.208$ \\
\hline D 87 & 0.0000 & $651874.8118,6835793.360$ \\
\hline V 88 & 0.0000 & $653042.9337,6835783.5004$ \\
\hline 89 & 0.0000 & $654209.7805,6835778.3790$ \\
\hline Total RMS error: & 0.0000 & \\
\hline
\end{tabular}

Fonte: Autor, 2016 
Segundo Sloan (2006, apud Lima, 2013, p. 51), um erro médio quadrático aceitável é igual ou menor que meio pixel e pode ser obtido através da equação abaixo, onde "M" é o módulo da escala da imagem e "R" a resolução da imagem em dpi. Inserindo-se na fórmula os valores utilizados resultaria:

$\mathrm{EMQ}_{\text {aceitável }}=[(0,0254 . \mathrm{M}) / \mathrm{R}] / 2=[(0,0254$. 10.000) $/ 200]=0,635$

Conforme a Figura 3 e a eq. 2 acima, verifica-se que o método utilizado superou as expectativas apresentando um erro médio quadrático total equivalente à $0 \mathrm{e}$, portanto, inferior ao EMQ aceitável.

A partir da verificação e da aceitação do RMS obtido, passou-se à etapa de vetorização. Na Figura 4 abaixo tem-se um recorte para visualização. Como o arquivo abrange varias feições, determinou um layer e uma cor para cada uma, de maneira que a diferenciação entre os elementos fosse facilitada.

Ao final deste processo o arquivo vetorial resultante, que neste caso possui o formato dwg, estará apto para ser complementado, editado e integrado com outros softwares, tanto de desenho como os que trabalham com Sistema de Informações Geográficas (SIG).

Figura 4 - Recorte do arquivo de vetorização com o mapa digitalizado ao fundo

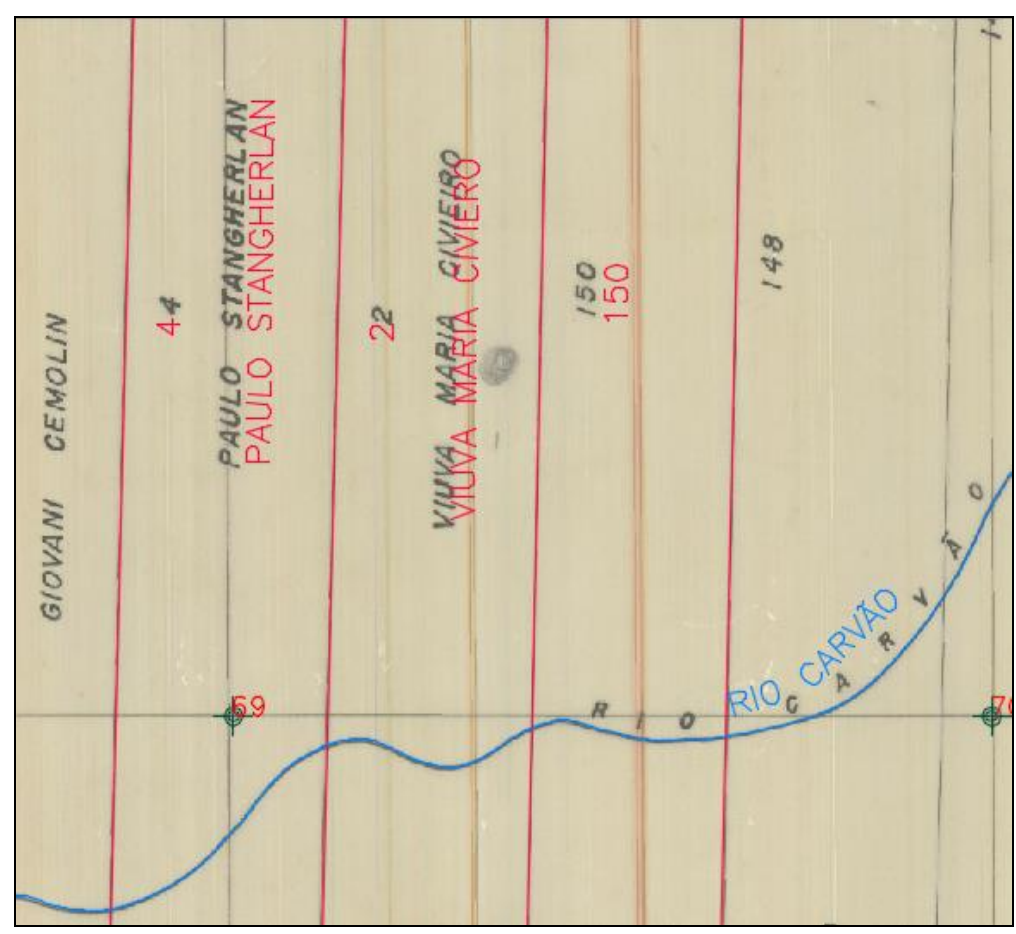

Fonte: Autor, 2016

\section{CONCLUSÃO}


Embora o desenvolvimento da cartografia atual se baseie sobretudo nas geotecnologias, é notável ainda a constante utilização de mapas antigos ou históricos nos mapeamentos, seja por guardarem os dados geográficos que são fundamentais para a reconstrução do passado, seja por possuírem informações que complementam os trabalhos atuais.

Com o intuito de tornar os mesmos reutilizáveis, através dos processos metodológicos apresentados no presente relatório, observou-se que é possível, de maneira relativamente simples, utilizar os mapas em formato analógico. Dentre a sequência apresentada composta por digitalização, edição da imagem, inserção do mapa no Autocad Map 3D, georreferenciamento e vetorização, constatou-se que a etapa que exige maior conhecimento técnico é justamente a penúltima. Portanto, sugere-se que os trabalhos futuros que venham a ser baseados no presente estudo, tenham um acompanhamento de um profissional da área de Geociências, para que os resultados obtidos possam ser confiáveis e reaproveitáveis.

Outro ponto a ser destacado é que embora o RMS tenha resultado zero, não se deve pensar equivocadamente que o mapa não possui erros. De fato, de acordo com Lima (2013, p. 31) “a imagem matricial resultante da digitalização do mapa original pode conter distorções oriundas de uma escanerização não paralela às bordas [...] ou da instabilidade do meio em que está", por exemplo variações de temperatura e umidade.

Levando-se em consideração os aspectos apresentados acima, conclui-se que o método utilizado para o georreferenciamento da planta no software Autocad Map 3D se mostrou eficaz para o alcance dos objetivos propostos e poderá servir de referência para trabalhos semelhantes, aumentando assim a utilização dos acervos das décadas passadas.

\section{REFERÊNCIAS}

CORRÊA, Douglas Corbari. Cartografia Histórica do Rio de Janeiro. 2008. 113 f. Tese (Doutorado) - Curso de Geografia, Universidade Federal do Rio de Janeiro, Rio de Janeiro, 2008.

OLIVEIRA, Fabíola Cech de. Técnica para o Estabelecimento de Plataforma Genética Utilizando Geoprocessamento. 2005. 79 f. TCC (Graduação) - Divisão de Engenharia de Infraestrutura Aeronáutica, Instituto Tecnológico da Aeronáutica, São José dos Campos, 2005. FILHO, Helius de Almeida Guimarães. Digitalização de Mapas. In: SIMPÓSIO BRASILEIRO DE SENSORIAMENTO REMOTO, 7, 1993, São José dos Campos. Anais... Campinas: Universidade Estadual de Campinas, 1993.8 p.

GIOTTO, E.; CARDOSO, C. D. V. Sobreposição de imagens georreferenciadas GIF no Google Earth com o sistema CR Campeiro 7. In: Semana da Geomática, 3, 2010, Santa Maria. Anais... Santa Maria: Colégio Politécnico da UFSM, 2010. p. 41-47.

LIMA, Erly Caldas de. O levantamento pioneiro da SARA Brasil: histórico, tecnologia empregada e avaliação dos produtos. 2012. Dissertação (Mestrado em Engenharia de Transportes) Escola Politécnica, Universidade de São Paulo, São Paulo, 2012. Disponível em: 
<http://www.teses.usp.br/teses/disponiveis/3/3138/tde-26072013-142950/>. Acesso em: 25 maio 2016.

MELO, Ivan Dornelas Falcone de; PACHÊCO, Admilson P. Aspectos da correção geométrica de imagens orbitais. In: Simpósio Brasileiro de Ciências Geodésicas e Tecnologias da Geoinformação, 1, 2004, Recife. Anais... Recife: Universidade Federal do Pernambuco, 2004.

NERO, Marcelo Antonio. Estudo Comparativo de Metodologias de Digitalização de Mapas e seu Controle de Qualidade Geométrica. 2000. 233 f. Dissertação (Mestrado) - Curso de Engenharia, Escola Politécnica da Universidade de São Paulo, São Paulo, 2000. Disponível em: <http://www.teses.usp.br/teses/disponiveis/3/3138/tde-24102005-145532/pt-br.php>. Acesso em: 25 abr. 2016.

SANTOS, Suzana Daniela Rocha; HUINCA, Suelen Cristina Movio; MELO, Lineardo Ferreira de Sampaio; DA SILVA, Marly Terezinha Quadri Simões; DELAZARI, Luciene Stamato. Considerações sobre a utilização do PEC (Padrão de Exatidão Cartográfica) nos dias atuais. In: SIMPÓSIO BRASILEIRO DE CIÊNCIAS GEODÉSICAS E TECNOLOGIAS DA GEOINFORMAÇÃO, 3, 2010, Recife. Anais... Recife: Universidade Federal de Paraná, 2010, 5 p. SILVA, Christian Nunes da; CARVALHO, João dos Santos. A representação espacial e a linguagem cartográfica. Geomae, Campo Mourão, v. 2, n. 2, p.85-106, set. 2011. 